\title{
Vaccination against 2009 pandemic H1N1 in a population dynamical model of Vancouver, Canada: timing is everything
}

\author{
Jessica M Conway ${ }^{1,2}$, Ashleigh R Tuite ${ }^{3}$, David N Fisman ${ }^{3}$, Nathaniel Hupert ${ }^{4,5}$, Rafael Meza', Bahman Davoudi ${ }^{1}$, \\ Krista English ${ }^{1}, \mathrm{P}$ van den Driessche ${ }^{6}$, Fred Brauer ${ }^{2}$, Junling Ma ${ }^{6}$, Lauren Ancel Meyers ${ }^{7}$, Marek Smieja ${ }^{8}$, \\ Amy Greer ${ }^{3,9}$, Danuta M Skowronski ${ }^{10}$, David L Buckeridge ${ }^{11,12}$, Jeffrey C Kwong ${ }^{3,13}$, Jianhong Wu ${ }^{14}$, \\ Seyed M Moghadas ${ }^{14}$, Daniel Coombs ${ }^{2}$, Robert C Brunham ${ }^{1}$ and Babak Pourbohloul ${ }^{1,15^{*}}$
}

\begin{abstract}
Background: Much remains unknown about the effect of timing and prioritization of vaccination against pandemic (pH1N1) 2009 virus on health outcomes. We adapted a city-level contact network model to study different campaigns on influenza morbidity and mortality.

Methods: We modeled different distribution strategies initiated between July and November 2009 using a compartmental epidemic model that includes age structure and transmission network dynamics. The model represents the Greater Vancouver Regional District, a major North American city and surrounding suburbs with a population of 2 million, and is parameterized using data from the British Columbia Ministry of Health, published studies, and expert opinion. Outcomes are expressed as the number of infections and deaths averted due to vaccination.

Results: The model output was consistent with provincial surveillance data. Assuming a basic reproduction number = 1.4, an 8-week vaccination campaign initiated 2 weeks before the epidemic onset reduced morbidity and mortality by $79-91 \%$ and $80-87 \%$, respectively, compared to no vaccination. Prioritizing children and parents for vaccination may have reduced transmission compared to actual practice, but the mortality benefit of this strategy appears highly sensitive to campaign timing. Modeling the actual late October start date resulted in modest reductions in morbidity and mortality (13-25\% and 16-20\%, respectively) with little variation by prioritization scheme.
\end{abstract}

Conclusion: Delays in vaccine production due to technological or logistical barriers may reduce potential benefits of vaccination for pandemic influenza, and these temporal effects can outweigh any additional theoretical benefits from population targeting. Careful modeling may provide decision makers with estimates of these effects before the epidemic peak to guide production goals and inform policy. Integration of real-time surveillance data with mathematical models holds the promise of enabling public health planners to optimize the community benefits from proposed interventions before the pandemic peak.

\section{Background}

The emergence of a novel swine-origin influenza A/ H1N1 virus in the spring of 2009 led the WHO to declare the first influenza pandemic of the 21st century [1]. In the Canadian province of British Columbia,

\footnotetext{
* Correspondence: babak.pourbohloul@bccdc.ca

'Division of Mathematical Modeling, University of British Columbia Centre for Disease Control, 655 West 12th Avenue, V5Z 4R4 Vancouver, British

Columbia, Canada

Full list of author information is available at the end of the article
}

Canada it first appeared as a spring-summer wave of very low intensity, but resurged as a more substantial and widespread second wave in the fall, as in the rest of Canada and many other countries worldwide $[2,3]$. The commencement of this second wave varied by jurisdiction, likely depending in part upon prior first wave experience, demographic and environmental factors. In British Columbia, second wave pandemic H1N1 (pH1N1) activity began slowly in early September 2009, coinciding with the reconvening of schools and

\section{() Biomed Central}


universities; increased more abruptly in mid-October; peaked during the last week of October; and fully resolved by the end of the calendar year [4]. This stands in marked contrast with Canada's usual influenza season, which typically spans November to April [5].

Characterization of the epidemiology of pH1N1 began early in the outbreak. One of the striking features of this novel influenza strain is its association with higher attack rates in younger individuals, compared to what is usually observed for seasonal influenza [6-12]. Proposed explanations for an apparently reduced susceptibility in older adults include pre-existing immunity due to prior exposure to related $\mathrm{H} 1 \mathrm{~N} 1$ strains circulating prior to 1957 [13], accumulated cross-protection derived from seasonal human H1N1 infection across the lifespan [14], and/or differences in the contact networks of younger individuals, who are more highly connected (and hence more likely to be exposed to pH1N1 early in an epidemic) than older individuals [15]. Regardless of the reasons underlying differential vulnerability to infection by age, this observation has important implications for the design and implementation of mitigation strategies for pH1N1 and future pandemic influenza viruses.

Vaccination is an important influenza control measure and was a key component of many countries' pandemic preparedness plans. Production of $\mathrm{pH} 1 \mathrm{~N} 1$ vaccine began soon after the pandemic potential of $\mathrm{pH} 1 \mathrm{~N} 1$ was recognized. However, the early arrival of the second wave of $\mathrm{pH} 1 \mathrm{~N} 1$ in many regions of the northern hemisphere, combined with production delays, resulted in the implementation of vaccination programs in populations already experiencing moderate to high incidence of pH1N1, a sequence of events expected to reduce the ultimate population impact of immunization. Quantifying this reduction and determining how it might have been mitigated through alternative dispensing schemes motivated the modeling effort reported here.

Seasonal influenza vaccination campaigns have historically targeted those at greatest risk of the severe outcomes of influenza-notably the very young, the elderly and individuals of all ages with underlying medical conditions, as well as their close contacts, including health care workers [5]. It has been suggested that vaccination of schoolchildren might be a more effective strategy [16], since younger age groups are responsible for a disproportionate amount of influenza transmission, and targeting these groups would indirectly protect at-risk groups $[17,18]$. Some regions-notably the province of Ontario-have adopted a universal influenza immunization program (UIIP) whereby influenza vaccine is provided free to all citizens over the age of 6 months [19]. In the case of $\mathrm{pH} 1 \mathrm{~N} 1$, the misalignment between vaccine availability and the onset and peak of the second pandemic wave in the fall 2009 required prioritization of vaccine. Greater infection risk and poor outcomes in younger individuals argued for targeted vaccination of younger age groups [18]. Conversely, older individuals were at decreased risk of infection with pH1N1, but experienced higher rates of severe outcomes, including mortality [20-22]. This is illustrated in outcome surveillance data from British Columbia for both waves, showing that $\mathrm{pH} 1 \mathrm{~N} 1$ detection was higher in children, but both the per-laboratory confirmed case hospitalization and fatality rates were greatest in older adults, with substantial increase beginning at age 50 . The $\mathrm{pH} 1 \mathrm{~N} 1$ vaccine prioritization schemes adopted by many countries required a balance of these competing considerations and ultimately differed from seasonal influenza recommendations as a result of vaccine delay and unique pandemic patterns of age-related risk [12,23-26].

Mathematical models of the spread of the pH1N1 virus across the population played a prominent role in the assessment of the $\mathrm{pH} 1 \mathrm{~N} 1$ pandemic risk and in the evaluation and design of intervention and control strategies. During the early stages of the pH1N1 pandemic, mathematical analyses of the initial data from Mexico and other countries allowed researchers to estimate the transmissibility of the $\mathrm{pH} 1 \mathrm{~N} 1$ virus, as measured by the basic reproduction number $[8,11,27,28]$. As the pandemic progressed, many modeling studies investigated the impact of different kinds of containment strategies like social distancing [29], vaccination [18,28-30], and the use of antivirals $[29,31]$. These studies among many others provided important information to policy makers and demonstrated the value of mathematical modeling as a risk assessment tool during the emergence of new infectious disease agents.

We developed a mathematical model of the transmission dynamics of the pH1N1 virus in the Greater Vancouver Regional District (GVRD) to quantify the impact of vaccination campaign timing in relation to the pandemic peak on the projected outcomes of these strategies. Models incorporating age structure and/or heterogeneity in disease vulnerability have long been used to investigate issues surrounding immunization, either with instantaneous vaccinations (e.g. [18,32-34]) or with vaccinations concurrent with the epidemic (e.g. [35]). In particular such models have been used to investigate vaccination strategies in combination with campaign timing, as in [36-38]. Our aim was to develop a population dynamical model that represents the transmission of pH1N1 influenza in a realistic urban setting. We therefore incorporated into this model detailed demographic and behavioural factors that provide the basis for pandemic transmission dynamics. In particular, in addition to modeling the age structure of the population, we also considered the heterogeneity in the contact rates between individuals by age to better represent the 
overall contact structure of the population and better approximate the time course of the epidemic. To parameterize our model we used data from the Greater Vancouver Regional District (GVRD), British Columbia $(B C)$, Canada. This model was used to assist policymakers in evaluating different intervention strategies throughout the Fall (2009) including the impact of vaccination of schoolchildren in addition to the specified target groups, social distancing, as well as assessing the likelihood of observing a third wave in the winter of 2010. However, as we discuss below, one should observe similar outcomes in most urban settings.

\section{Methods}

\section{Model overview}

We developed a susceptible-infected-recovered (SIR)-type compartmental model, extended to capture heterogeneity in age and behaviour, both of which affect contact patterns between individuals. A complete description of the model is provided in the Appendix, Additional file 1. The population was divided into six compartments representing different disease states: susceptible $(\mathrm{S})$, vaccinated against pH1N1 (SW), exposed (E), pre-symptomatically infectious (before clinical infection onset) (A), infectious (either symptomatic, after clinical infection onset; or asymptomatic) (I), and immune (M). In an attempt to capture the social network-type dynamics in a more computationally tractable manner, the population was stratified by age and activity level. Activity level groupings correspond to the average number of contacts that individuals have per week. Mixing of the different age/activity groups was obtained from models of the GVRD contact network $[39,40]$. Each compartment is comprised of many subcompartments for each age and activity level grouping. We ran the model to investigate the time period from September 1, 2009 until May 31, 2010.

\section{Model parameterization}

Epidemiological parameters for pH1N1 were derived from the published literature and empirical data, with some assumptions reflecting the nature of influenza infection (Table 1). For simulations, the baseline transmission parameter values were: basic reproduction number $R_{0}$ of 1.4 , latent period of 3 days, and infectious period of 7 days ( 1 day before clinical infection onset and 6 days either symptomatic, after clinical infection onset, or asymptomatic) [21,41]. Demographic and behavioural data used to derive age-specific average number of contacts per week was obtained for the GVRD $[39,42]$.

\section{Initial condition}

We set the start time of the epidemic to September 6th, which corresponds to the start of school in Vancouver.
As of August 31st there had been only a total of 812 laboratory-confirmed cases of pH1N1 influenza since April of 2009 in British Columbia [48]. We assumed that the number of actual (currently infected) cases on September 6th was 100 . We then distributed them through the age and behaviour compartments randomly with probably weighted by population fraction and contact rate in each compartment. Each result we show in the following represents the mean of 10000 simulations starting with different random initial conditions. We further assume that the rest of the population is completely susceptible. Although there was $\mathrm{pH} 1 \mathrm{~N} 1$ activity in the GVRD in Spring 2009 which would result in some background immunity, it was quite low, as evidenced by numbers of laboratory-confirmed cases and reported hospitalizations [48]. We therefore assumed the effect of background immunity was negligible.

\section{Vaccination implementation}

The time to administer vaccine across the population was assumed to be 8 weeks. Vaccine distribution spanned this roll-out period and resulted in final coverage levels in different age groups (described below). For results shown below, we assumed the daily number of vaccinations gradually decreased throughout the campaign. However using different vaccination rates gave quantitatively and qualitatively similar results; see Appendix, Additional file 1 for details and additional information. We assumed that there was no intra-group age prioritization for vaccine distribution among those who were eligible to receive the vaccine. We assumed a 2 -week delay between vaccine receipt and development of a protective immune response [49].

All individuals receiving vaccine were assumed to have a reduction in pH1N1 acquisition risk equal to $90 \%$ (modeled as a "leaky vaccine"). Although this number may seem high at first, studies of both the efficacy and the effectiveness of the pH1N1 vaccine used in Canada have shown remarkably high levels of protection $[45,46,50]$. In particular, initial studies of seroconversion and seroprotection rates by the adjuvanted $\mathrm{pH} 1 \mathrm{~N} 1$ vaccine used in Canada showed high levels $(>90 \%)$ of both consistently across all age groups $[45,46]$. Further, a recent study with over 500 participants in Canada showed that this vaccine was highly effective at preventing laboratory-confirmed $\mathrm{pH} 1 \mathrm{~N} 1$ influenza [50]. The reported high vaccine protection (effectiveness), generally over $90 \%$, was maintained across most sensitivity analyses [50]. These results are supported by a similar study investigating the effectiveness of a the same vaccine in children $<10$ years of age, which reported statistically significant $100 \%$ vaccine effectiveness for a single dose assuming a 2-week development of protective immune response [51]. We therefore assumed a baseline 
Table 1 Model parameter values

\begin{tabular}{|c|c|c|c|}
\hline Variable & $\begin{array}{l}\text { Age } \\
\text { group }\end{array}$ & $\begin{array}{l}\text { Value } \\
\text { (range) }\end{array}$ & Source \\
\hline \multirow[t]{8}{*}{ Population size } & $0-2$ & 63,025 & 2006 Census [42] \\
\hline & $3-4$ & 42,260 & \\
\hline & $5-17$ & 322,670 & \\
\hline & $18-24$ & 203,500 & \\
\hline & $25-54$ & 975,875 & \\
\hline & $55-64$ & 237,795 & \\
\hline & $\geq 65$ & 271,455 & \\
\hline & Total & $2,116,580$ & \\
\hline Latent period (days) & All & $3(2-4)$ & Tuite et al. 2010 [22] \\
\hline $\begin{array}{l}\text { Initial asymptomatic infectious period (in all infected } \\
\text { individuals) (days) }\end{array}$ & All & 1 & Liao et al. 2010 [43] \\
\hline Total duration of infectiousness (days) & All & $7(5-7)$ & Tuite et al. 2010 [22]; De Serres [44] \\
\hline Basic Reproduction number $\left(R_{0}\right)$ & All & $1.4(1.2-1.8)$ & Pourbohloul et al. 2009 [27] \\
\hline Proportion of population with pre-existing immunity & $\geq 55$ & 0.5 & $\begin{array}{l}\text { Centers for Disease Control and Prevention } 2009 \text { [13]; Fisman } \\
\text { et al. } 2009 \text { [6] }\end{array}$ \\
\hline Vaccine efficacy & All & $0.9(0.5-1)$ & $\begin{array}{l}\text { Product Monograph Arepanrix(tm) H1N1 [45]; Waddington et } \\
\text { al., } 2010 \text { [46] }\end{array}$ \\
\hline Proportion of infected individuals who self-isolate & All & $0.1(0.1-0.6)$ & Assumption \\
\hline \multirow[t]{7}{*}{ Mortality (per 100,000 infections) } & $0-2$ & 30 & Donaldson et al. 2009 [47] \\
\hline & $2-4$ & 27 & \\
\hline & $5-17$ & 11 & \\
\hline & $18-24$ & 12 & \\
\hline & $25-54$ & 30 & \\
\hline & $55-64$ & 65 & \\
\hline & $\geq 65$ & 980 & \\
\hline
\end{tabular}

vaccine efficacy/effectiveness of 0.9 across all age groups. Although as mentioned here there is strong evidence that the protection offered by the $\mathrm{pH} 1 \mathrm{~N} 1$ vaccine predominantly distributed in Canada was extremely high, we nevertheless also performed extensive sensitivity analyses of our results and conclusions assuming much lower values of vaccine protection (see Figures S5 and S6, Additional file 1).

\section{Vaccination scenarios}

We considered four different vaccination strategies. In the first two and fourth scenarios, the final population vaccination coverage was approximately $47 \%$, matching age-standardized estimates of overall $\mathrm{pH} 1 \mathrm{~N} 1$ vaccine coverage in the GVRD. However, each of these scenarios simulated different patterns of vaccine distribution across age groups. The Actual Coverage (AC) strategy assumed a vaccine uptake in the different age groups corresponding to the observed uptake of $\mathrm{pH} 1 \mathrm{~N} 1$ vaccine in the GVRD during the Fall vaccination campaign of 2009 , which covered an aggregate of $47 \%$ of the population (BC Centre for Disease Control, personal communication; see Table 2 for age-specific vaccination coverage). For the Uniform Coverage (UC) strategy, final vaccine uptake was set at $47 \%$ within each age group.

Because of heightened interest in prioritization of demographic groups potentially capable of accelerating early transmission of influenza [18], we also modeled a Parents and Children (PC) strategy in which $100 \%$ of children aged 5-17 and their parents (represented by $100 \%$ of adults aged $30-39$ ) received the vaccine. No other members of the population were vaccinated under this strategy, which had a final population coverage of only $36 \%$ in contrast to the $47 \%$ of the first two scenarios, a difference that corresponds to over 230,000 fewer doses distributed throughout the GVRD.

Finally since the PC strategy has a lower population coverage than the first two, we formulated a fourth strategy that combines it with a more general vaccine distribution to attain a final $47 \%$ coverage. In the AC and UC coverage scenarios described above, $36 \%$ population coverage is achieved in approximately 38 days. For this fourth scenario, the parents-and-children/actual sequence strategy $(\mathrm{PC}+)$, we assume that after that point (38 days) the vaccine is then made available to the 
Table 2 Age-specific coverage levels for the different vaccination scenarios

\begin{tabular}{|c|c|c|}
\hline Vaccination strategy & Age group & Vaccination coverage (\%) \\
\hline \multirow[t]{8}{*}{ Actual pH1N1 vaccination in the Greater Vancouver Regional District (AC) } & $0-2$ & 60.0 \\
\hline & $3-4$ & 60.0 \\
\hline & $5-17$ & 49.0 \\
\hline & $18-24$ & 36.1 \\
\hline & $25-54$ & 41.4 \\
\hline & $55-64$ & 47.4 \\
\hline & $\geq 65$, community-dwelling & 58.8 \\
\hline & $\geq 65$, long-term care & 58.8 \\
\hline Uniform coverage (UC) & All ages & 47 \\
\hline \multirow[t]{3}{*}{ Parents and children (PC) } & $5-17$ & 100 \\
\hline & 30-39 & 100 \\
\hline & All other ages & 0 \\
\hline \multirow[t]{8}{*}{ Parents and children/actual sequence (PC+) } & $0-2$ & 35.0 \\
\hline & $3-4$ & 35.0 \\
\hline & $5-17$ & 100.0 \\
\hline & $18-24$ & 24.9 \\
\hline & $25-54$ & 33.8 \\
\hline & $55-64$ & 27.6 \\
\hline & $\geq 65$, community-dwelling & 34.3 \\
\hline & $\geq 65$, long-term care & 34.3 \\
\hline
\end{tabular}

general public for the remaining time of the campaign. We model this using AC coverages scaled down proportionally to make up for the $11 \%$ deficit in coverage of the PC strategy.

To reflect actual pH1N1 response activities in GVRD, we initiated each of the modeled vaccination programs on October 26, 2009. For the baseline 8-week campaign length, vaccination was completed by the end of the week of December 14, 2009.

\section{Sensitivity analyses}

We tested the robustness of projections to model assumptions by performing sensitivity analyses over plausible ranges of parameter values. A range of values for $R_{0}$, latent period, infectious period, vaccine efficacy, and vaccination campaign lengths (see Table 1) were tested in the absence of vaccination (where appropriate) and in the presence of each of the three vaccination strategies. For each vaccination strategy, we also tested the effect of varying the start date of vaccination campaigns under baseline transmission parameter values. Model outputs were assessed for vaccination campaigns initiated at the beginning of each week from July 5 , 2009, to November 22, 2009. Finally, we also assessed the impact of using different $\mathrm{pH} 1 \mathrm{~N} 1$ age-specific mortality profiles on our results.

\section{Results}

\section{Baseline case without and with vaccination}

Although true pH1N1 infection incidence is difficult to determine, the recorded spread of $\mathrm{pH} 1 \mathrm{~N} 1$ through different age groups in the GVRD starting in the early autumn of 2009 was closely reproduced by the model using the baseline parameter values for pH1N1 (Figure 1 ). In particular, the model predicted that the highest number of infections in the 18-54 age group, followed by the $5-17,0-4$, and $\geq 55$ age groups (Figure $1 \mathrm{~d}$ ), which is similar to what was observed within laboratory-confirmed reported cases. Further, the model predicted a peak of pH1N1 activity in Vancouver in early November and highest age-specific attack rates in the 5-17 age group, followed by the $18-54,0-4$, and $\geq 55$ age groups (Figure 2, dashed lines).

Figure 2 shows the impact of simulating the actual GVRD pH1N1 vaccination campaign to the baseline model (Actual Coverage strategy initiated October 26, 2009, dashed lines). This intervention reduced the simulated cumulative attack rate from $48.3 \%$ to $42.0 \%$, representing over $120,000 \mathrm{pH} 1 \mathrm{~N} 1$ infections prevented in the Vancouver population. The number of cases prevented is greatest in the 5-17 year old age group (7625 per 100,000 population), followed by the 18-54 (6011 per $100,000)$, 0-4 (5824 per 100,000$)$, and $\geq 55$ (4152 per 


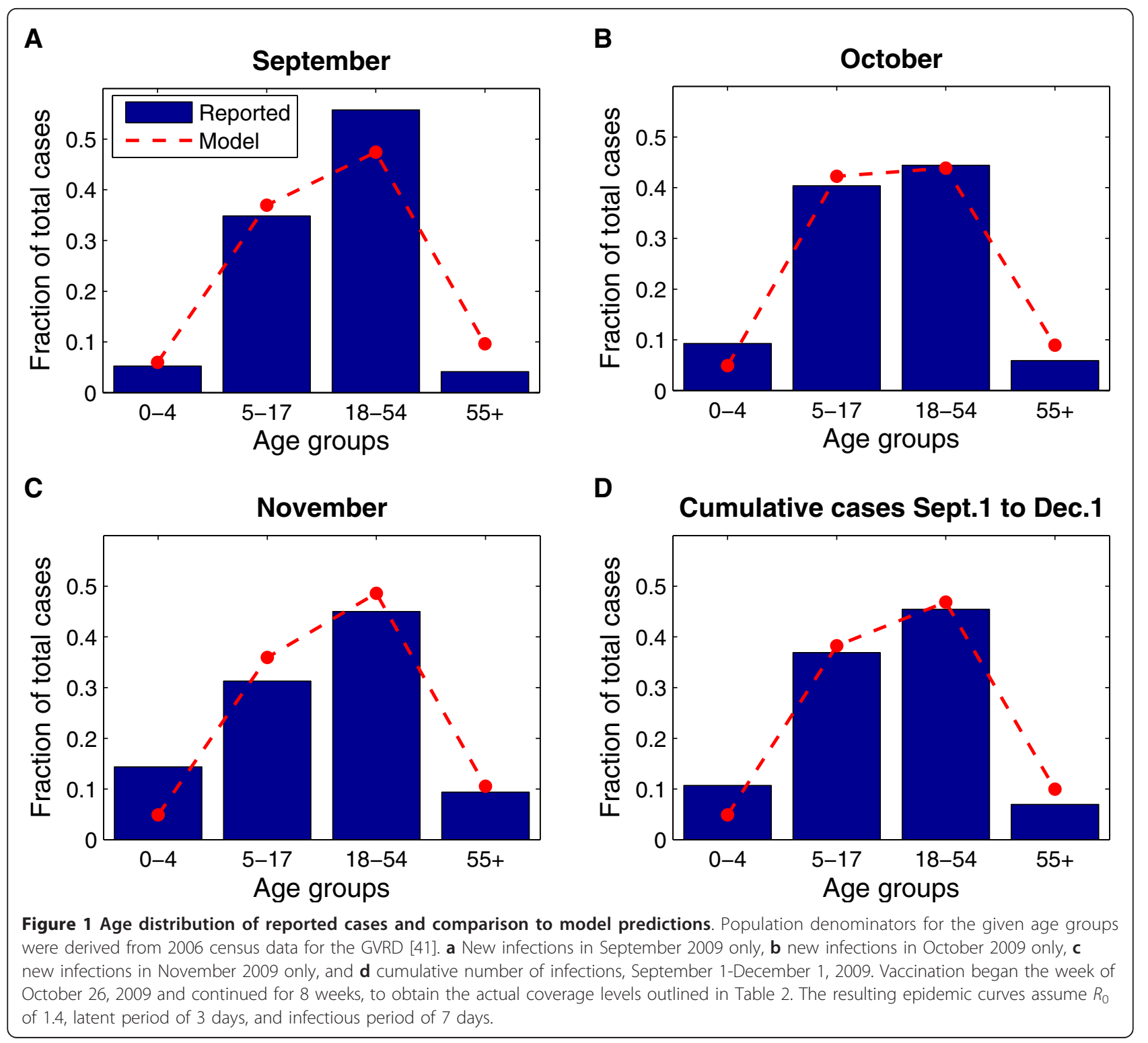

$100,000)$ age groups. The fraction of cases prevented is not equivalent across age groups with this strategy: individuals aged $0-4$ and $\geq 55$ years experience the largest relative reduction in final attack rate (18\% decrease), while those in the 5-17 age group have the smallest (10\% decrease).

Impact of timing of vaccination campaign on final attack rates

As expected, earlier implementation of the Actual Coverage strategy resulted in smaller final attack rates (Figure 3). Initiation of vaccination campaigns in the presence of moderate levels of circulating $\mathrm{pH} 1 \mathrm{~N} 1$, but prior to the epidemic peak, had a modest but detectable impact on final attack rates. For example, under baseline assumptions, an 8-week campaign initiated 2 weeks before epidemic onset (August 24) reduced the attack rate by approximately $83 \%$; an 8 -week campaign initiated 1 month into the epidemic (October 5) reduced the attack rate by approximately $47 \%$. Additionally, distribution of vaccine in a shorter period of time resulted in a greater reduction in attack rates for a given vaccination campaign start date.

Transmission and mortality impact of different vaccination strategies

Under baseline assumptions (i.e., vaccination initiated on October 26, 2009 with an 8-week campaign), vaccination of not only parents and children/general population in sequence $(\mathrm{PC}+)$, but also of parents and children $(\mathrm{PC})$ 


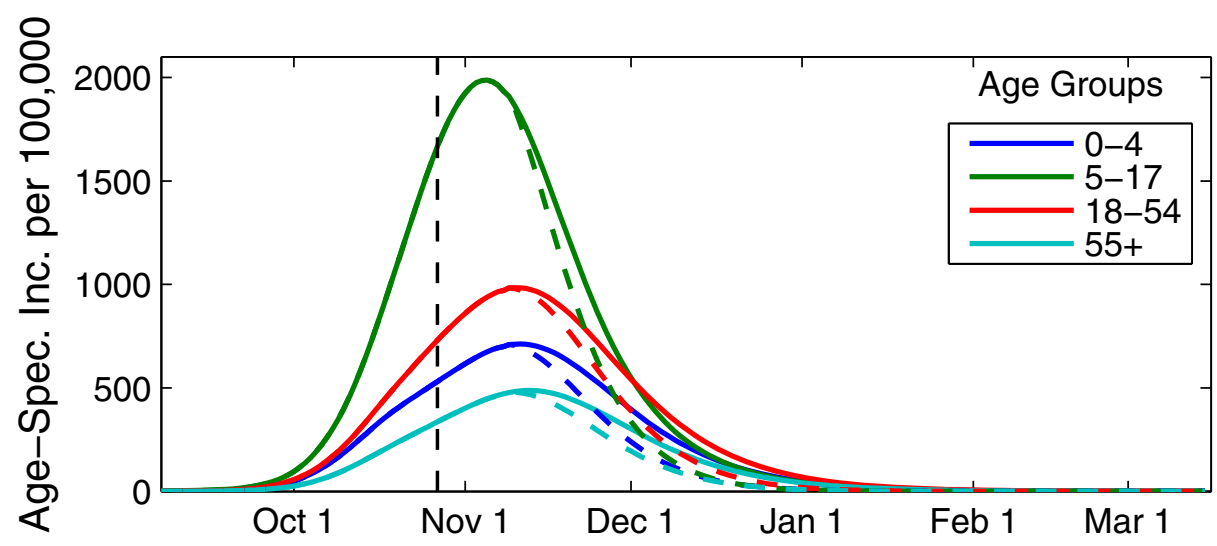

Figure 2 Age-specific daily incidence of pH1N1 cases. The number of new cases per day per 100,000 individuals is presented in the absence (solid lines) and presence (dotted lines) of pH1N1 vaccination. Vaccination began the week of October 26, 2009 and continued for 8 weeks, to obtain the actual coverage levels outlined in Table 2. The resulting epidemic curves assume $R_{0}$ of 1.4, latent period of 3 days, and infectious period of 7 days.

alone were more effective than the actual coverage (AC) or a uniform coverage (UC) strategy in reducing the influenza attack rate but each achieved equivalent mortality reduction (see Tables 3 and 4, Figure $4 \mathrm{a}$ and $4 \mathrm{~b}$, and Figures $\mathrm{S} 1$ and S2, Additional file 1 for additional values of $R_{0}$ ). Comparing the $\mathrm{AC}$, UC PC, and $\mathrm{PC}+$ strategies for different campaign initiation end times yielded more complex results. Prior to the start of the fall wave (August 24, 2009 or earlier), the PC strategy resulted in lower attack rates and mortality in the protected age groups (5-17 and 18-54) but higher attack rates and mortality in the other age groups $(0-4$ and $\geq 55)$ than either the AC or the UC strategies. The PC+ strategy yielded lower still attack rates but also the lowest overall mortality reduction. For campaigns initiated during the fall wave but prior to the epidemic peak (September 28 and October 26, respectively), the PC and PC+ strategies were superior in both attack rate and mortality reductions. During this same time frame the AC strategy was more successful at reducing attack rates than the UC strategy, with a minor exception in the 18-54 age group for which the UC strategy was favorable. This general trend likely resulted from higher AC coverage in the age groups with both the highest age-specific attack rates (5-17 year olds) and the most vulnerable age groups (04 year olds and $\geq 65$ ). This explanation is supported by

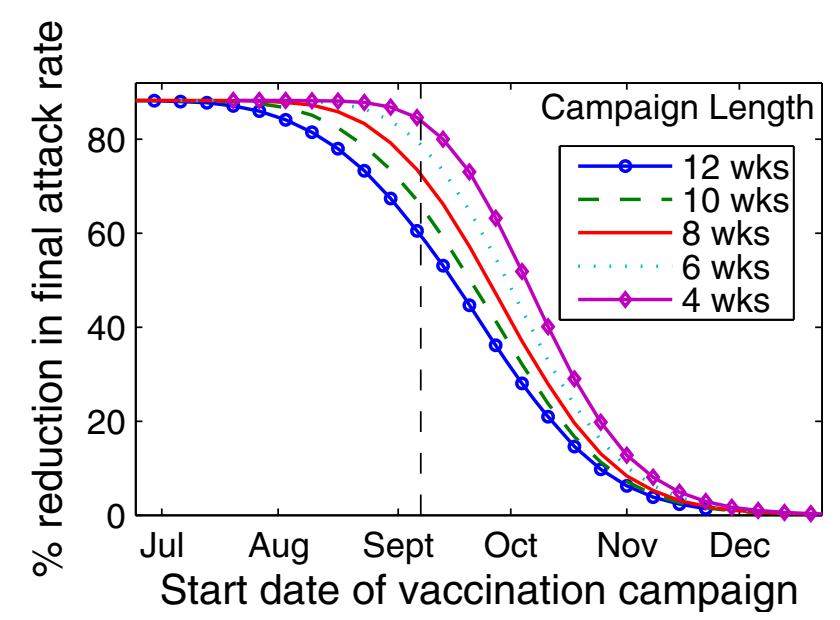

Figure 3 Effect of vaccination campaign start date on overall attack rate. For a given vaccination campaign start date, the percent reduction in final attack rate relative to that observed in the absence of vaccination is presented for campaign lengths of between 4 and 12 weeks. Vaccination campaigns were implemented weekly, starting July 5, 2009, with the last campaign started November 22, 2009. The start of the Vancouver influenza season on September 6, 2009 is indicated by a vertical line. All simulations assumed $R_{0}$ of 1.4, latent period of 3 days, and infectious period of 7 days. 
Table 3 Overall and age-specific final attack rates for pH1N1 for different vaccination scenarios for $R_{0} 1.4$ and an 8 week vaccine campaign length.

\begin{tabular}{|c|c|c|c|c|c|c|}
\hline \multirow[t]{2}{*}{ Vaccination start date } & \multirow[t]{2}{*}{ Vaccination strategy } & \multicolumn{5}{|c|}{ Attack rate,\% (\% reduction vs. None) } \\
\hline & & All ages & $0-4$ & $5-17$ & $18-54$ & $\geq 55$ \\
\hline None & None & 48.3 & 35.1 & 78 & 47.5 & 24.1 \\
\hline \multirow[t]{4}{*}{ 24-Aug } & $A C$ & $8.0(83)$ & $4.6(87)$ & $12.0(85)$ & $8.9(81)$ & $3.2(87)$ \\
\hline & $\overline{U C}$ & $10.1(79)$ & $6.7(81)$ & $20.0(74)$ & $8.8(81)$ & $4.0(83)$ \\
\hline & $\mathrm{PC}$ & $6.1(87)$ & $7.8(78)$ & $1.1(99)$ & $8.5(82)$ & $5.0(79)$ \\
\hline & $\mathrm{PC}+$ & $4.3(91)$ & $5.0(86)$ & $0.9(99)$ & $6.2(87)$ & $3.2(87)$ \\
\hline \multirow[t]{4}{*}{ 28-Sep } & $A C$ & $25.5(47)$ & $16.0(54)$ & $42.3(46)$ & $25.7(46)$ & $10.9(55)$ \\
\hline & UC & $26.8(44)$ & $18.3(48)$ & $49.2(37)$ & $24.7(48)$ & $11.7(51)$ \\
\hline & PC & $14.1(71)$ & $14.0(60)$ & $9.4(88)$ & $18.3(62)$ & $9.5(61)$ \\
\hline & $\overline{P C+}$ & $12.1(75)$ & $12.3(65)$ & $7.7(90)$ & $15.9(67)$ & $8.2(66)$ \\
\hline \multirow[t]{4}{*}{$26-O c t$} & $A C$ & $42.0(13)$ & $28.9(18)$ & $69.8(10)$ & $41.1(13)$ & $19.7(18)$ \\
\hline & $\mathrm{UC}$ & $42.0(13)$ & $29.8(15)$ & $71.5(8)$ & $40.3(15)$ & $19.9(17)$ \\
\hline & $P C$ & $37.7(22)$ & $29.3(16)$ & $54.1(31)$ & $39.3(17)$ & $20.1(17)$ \\
\hline & $\mathrm{PC}+$ & 36.4 (25) & 28.5 (19) & $52.0(33)$ & $38.0(20)$ & $19.5(19)$ \\
\hline \multirow[t]{4}{*}{ 23-Nov } & $A C$ & $47.4(2)$ & 34.1 (3) & $77.1(1)$ & $46.5(2)$ & $23.4(3)$ \\
\hline & $\underline{U C}$ & $47.4(2)$ & $34.2(2)$ & $77.2(1)$ & $46.4(2)$ & $23.4(3)$ \\
\hline & $P C$ & 47.0 (3) & $34.4(2)$ & $75.2(4)$ & $46.5(2)$ & $23.6(2)$ \\
\hline & $\overline{\mathrm{PC}+}$ & 46.8 (3) & $34.3(2)$ & $74.9(4)$ & $46.3(3)$ & $23.5(2)$ \\
\hline
\end{tabular}

Attack rate is defined here as the total number of infections. 'AC' indicates the actual vaccination coverage for $\mathrm{pH} 1 \mathrm{~N} 1 \mathrm{in}$ the GVRD, 'UC' the uniform coverage vaccination strategy, ' $\mathrm{PC}$ ' the parents and children vaccination strategy, and ' $\mathrm{PC}+$ ' the $\mathrm{PC} /$ actual sequence strategy

Table 4 Overall and age-specific pH1N1-attributable mortality for different vaccination scenarios for $\boldsymbol{R}_{\mathbf{0}} \mathbf{1 . 4}$ and an 8week vaccine campaign length.

\begin{tabular}{|c|c|c|c|c|c|c|}
\hline \multirow[t]{2}{*}{ Vaccination start date } & \multirow[t]{2}{*}{ Vaccination strategy } & \multicolumn{5}{|c|}{ Mortality per 100,000 population (\% reduction vs. None) } \\
\hline & & All ages & $0-4$ & $5-17$ & $18-54$ & $\geq 55$ \\
\hline None & None & 29.6 & 8.6 & 7.4 & 10.9 & 101.1 \\
\hline \multirow[t]{4}{*}{ 24-Aug } & AC & $4.1(86)$ & $1.1(87)$ & $1.1(85)$ & $2.1(81)$ & $12.7(87)$ \\
\hline & $\underline{U C}$ & $5.2(82)$ & $1.6(81)$ & $1.9(74)$ & $2.0(81)$ & $17.1(83)$ \\
\hline & $P C$ & $5.8(80)$ & $1.9(78)$ & $0.1(99)$ & $1.9(83)$ & $21.7(79)$ \\
\hline & $\mathrm{PC}+$ & $3.7(87)$ & $1.2(86)$ & $0.1(99)$ & $1.4(87)$ & $13.5(87)$ \\
\hline \multirow[t]{4}{*}{ 28-Sep } & $A C$ & $13.7(54)$ & $3.9(54)$ & $4.0(46)$ & $6.0(46)$ & $44.4(56)$ \\
\hline & UC & $14.9(50)$ & $4.5(48)$ & $4.6(37)$ & 5.7 (48) & $49.4(51)$ \\
\hline & $P C$ & $11.3(62)$ & $3.4(60)$ & $0.9(88)$ & $4.2(62)$ & $40.5(60)$ \\
\hline & $\mathrm{PC}+$ & $9.8(67)$ & $3.0(65)$ & $0.7(90)$ & $3.6(67)$ & $35.1(65)$ \\
\hline \multirow[t]{4}{*}{ 26-Oct } & $\underline{A C}$ & $24.4(18)$ & $7.1(18)$ & $6.6(10)$ & 9.5 (13) & 81.7 (19) \\
\hline & UC & $24.8(16)$ & $7.3(15)$ & $6.7(8)$ & 9.3 (15) & $83.8(17)$ \\
\hline & $P C$ & $24.5(17)$ & $7.2(16)$ & $5.1(31)$ & 9.0 (18) & $84.6(16)$ \\
\hline & $\mathrm{PC}+$ & $23.7(20)$ & $7.0(19)$ & $4.9(33)$ & $8.7(20)$ & $82.0(19)$ \\
\hline \multirow[t]{4}{*}{ 23-Nov } & $A C$ & 28.7 (3) & $8.4(3)$ & $7.3(1)$ & $10.7(2)$ & 97.9 (3) \\
\hline & UC & $28.8(3)$ & $8.4(2)$ & $7.3(1)$ & $10.7(2)$ & $98.2(3)$ \\
\hline & $P C$ & $29.0(2)$ & $8.5(2)$ & $7.1(4)$ & $10.7(2)$ & $99.2(2)$ \\
\hline & $\mathrm{PC}+$ & $28.9(2)$ & $8.4(2)$ & $7.1(4)$ & 10.7 (3) & $98.8(2)$ \\
\hline
\end{tabular}

Attack rate is defined here as the total number of infections. 'AC' indicates the actual vaccination coverage for pH1N1 in the GVRD, 'UC' the uniform coverage vaccination strategy, ' $U C$ ' the uniform coverage vaccination strategy, ' $P C$ ' the parents and children vaccination strategy, and ' $P C+$ ' the $P C / a c t u a l$ sequence strategy 


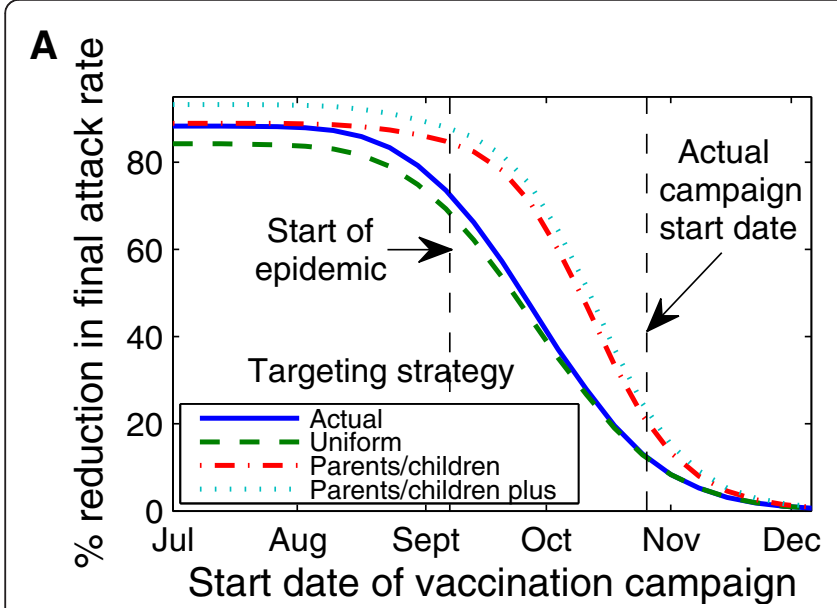

B

Figure 4 Impact of timing on the effectiveness of different vaccination strategies. Vaccination campaigns were implemented weekly, starting July 5, 2009, with the last campaign started November 22, 2009. For a given campaign start date, the reduction in a final attack rates and $\mathbf{b}$ mortality relative to no vaccination was assessed using actual (blue, solid line), uniform (green, dashed line), parents and children only (red, dash-dotted line), or parents and children only/actual sequence (cyan, dotted line) vaccination strategies. All simulations assumed $R_{0}$ of 1.4 , latent period of 3 days, infectious period of 7 days and an 8-week vaccine roll-out period.

the overall success, in both attack rate reduction and mortality reduction, of the $\mathrm{PC}+$ strategy: as with the $\mathrm{PC}$ strategy, the age group with highest age-specific attack rates is completely protected, but there is also limited protection for the most vulnerable age groups. There were no substantive differences in the outcomes of all vaccination strategies $(\mathrm{AC}, \mathrm{UC}, \mathrm{PC}$, or $\mathrm{PC}+$ ) when the campaigns were initiated well after the epidemic peak (on November 23).

These results are especially notable because of the difference in the overall coverage between the strategies: $47 \%$ for the AC, UC, and PC+ strategies vs $36 \%$ for the PC strategy. Similar results were observed for pandemic viruses exhibiting less transmissibility than pH1N1 (e.g., $R_{0}=1.2$ ) (see Figures $\mathrm{S} 1$ and S2, Additional file 1 and Tables S1-S6, Additional file 1 for additional values of $R_{0}$ ), assuming different reported age-specific mortality profiles (see Figure S3, Additional file 1), and considering only the PC strategy assuming lower coverage levels in the parents and children groups (see Figure S4, Additional file 1).

\section{Vaccine efficacy}

We evaluated the interplay between $\mathrm{pH} 1 \mathrm{~N} 1$ vaccine efficacy and the timing of the vaccination campaign for the Actual Coverage strategy (Figure 5, see Figure S5, Additional file 1 for sensitivity of cumulative attack rate to vaccine efficacy). If the simulated campaign begins well before the onset of the epidemic, vaccine efficacy was observed to have an important impact on depleting the size of the susceptible population and consequently reducing the outbreak size. For a vaccine with $85 \%$ or $95 \%$ efficacy, for example, the percent reduction in final attack rate relative to that observed in the absence of vaccination was $85 \%$ or $92 \%$, respectively. However, for vaccination campaigns initiated after the onset of the epidemic, reductions in final attack rates were not highly sensitive to vaccine efficacy. For example, when vaccinations were implemented late in the epidemic stage (October or November) the percent reduction in final attack rate varied only slightly when vaccine efficacy increased from $50 \%$ to $100 \%$ (almost vertical lines representing the $5 \%$ and $15 \%$ contours in Figure 5). We observed similar patterns for other coverage scenarios (see Figure S6, Additional file 1).

\section{Sensitivity of results to transmission parameters for pH1N1}

Varying epidemiological parameters changed the cumulative attack rate in the presence of the Actual Coverage strategy in predictable ways. This is clear from Figure 6 (see Figure S7, Additional file 1 for results in the absence of vaccination), where we show a sensitivity analysis on the cumulative attack rate for a given $R_{0}$. To generate the shaded areas, we ran simulations for parameter combinations from the ranges given in Table 1 with each combination given equal wait. We observed in particular that the lengths of shortening the infectious and latent periods resulted in more rapid epidemic growth and larger final outbreak sizes for a given value of $R_{0}$, due to the shorter window of opportunity for vaccination to have an effect. Lengthening these parameters had the opposite effect. The sensitivity of final attack rates to the latent and infectious periods diminished as transmissibility (represented by $R_{0}$ ) increased. 


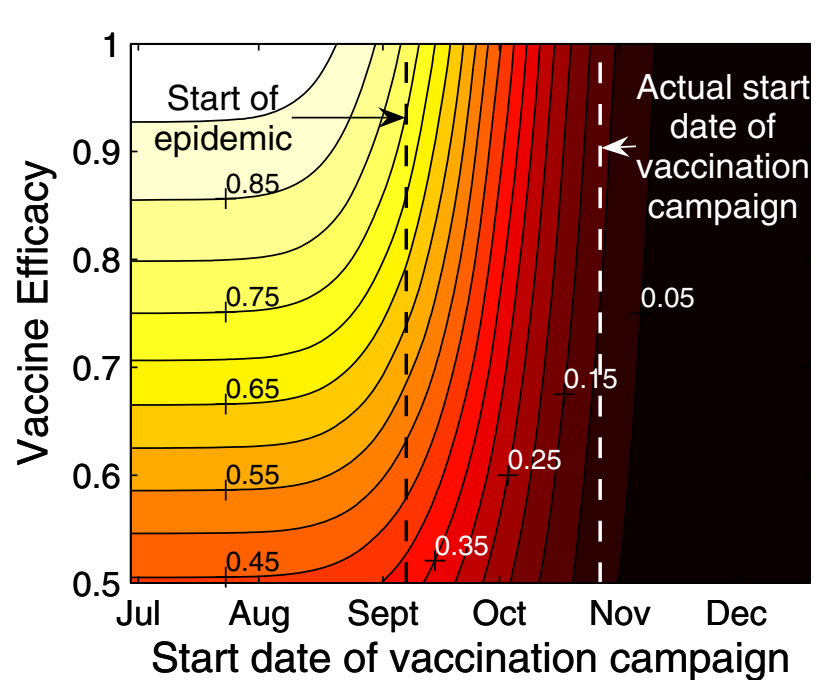

Figure 5 Effect of vaccination campaign start date and vaccine efficacy on overall attack rates. For a given vaccination campaign start date, the percent reduction in final attack rate relative to that observed in the absence of vaccination is presented for vaccine efficacy of between $50 \%$ and $100 \%$. The ranges of the percent reduction in attack rates are indicated by solid lines and labeled. Vaccination campaigns were implemented weekly, starting July 5, 2009, with the last campaign started November 22, 2009. The start of the Vancouver influenza season on September 6, 2009 is indicated by a vertical line. All simulations assumed $R_{0}$ of 1.4, latent period of 3 days, and infectious period of 7 days.

\section{Discussion}

Using detailed demographic information for the GVRD, we have developed a compartmental mathematical model to estimate the transmission of $\mathrm{pH} 1 \mathrm{~N} 1$ in this population and to examine the impact of timing and age-specific coverage of different vaccination strategies for reducing the disease burden of pH1N1. Our simulations and sensitivity analyses uncovered findings with significant public health implications. First, we quantified the effect of delay in vaccine distribution relative to levels of pandemic influenza virus circulation in the population. Although vaccination is a well-established influenza preventive measure, we showed that its effectiveness during a pandemic depends greatly on the capacity to produce, distribute, and dispense vaccine in a timely manner. We demonstrated as well the importance of considering the interplay between vaccine campaign timing, demographics (especially age-specific

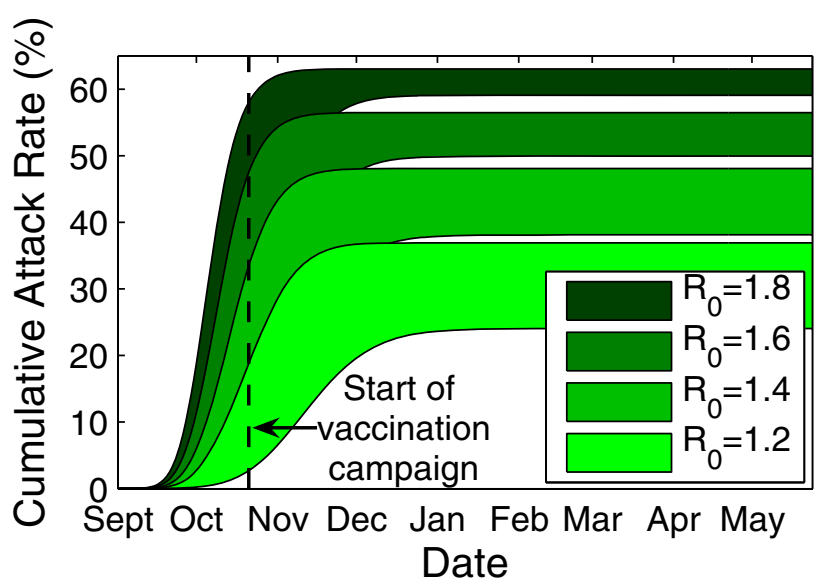

Figure 6 Sensitivity of cumulative attack rates to epidemiological parameters. Cumulative attack rates were determined for a range of values of $R_{0}(1.2-1.8)$, latent period (2-4 days), and infectious period (5-7 days) in the presence of pH1N1 vaccination. The range of results observed for varying latent and infectious period lengths are presented for each value of $R_{0}$. Vaccination began the week of October 26, 2009 and continued for 8 weeks, to obtain the actual coverage levels outlined in Table 2 . For a given value of $R_{0}$, the most steep curves (and highest attack rates in the presence of vaccination) were observed with a latent period of 2 days and infectious period of 5 days, and the least steep curves (lowest attack rates with vaccination) were observed with a latent period of 4 days and infectious period of 7 days. 
contact rates), and the epidemiologic characteristics of the disease when developing vaccination strategies. Our sensitivity analyses verified the robustness of the results reported herein, despite the necessary inclusion of parameters in our model for which accurate estimates are currently non-existent.

We included population activity levels in our mathematical model based on a realistic representation of the contact network in the GVRD. We believe that this substantially improves the realism of the model, and gives us greater confidence in our results. For example, certain small subpopulations (e.g. health care workers or children) can have a large number of potentially diseasetransmitting contacts per week, and are therefore more likely to acquire and transmit infection. Our model captures this important effect, while simplified models with homogenized activity levels would not. Age groupings addressed age-related variations in $\mathrm{pH} 1 \mathrm{~N} 1$ vulnerability to infection versus severe outcomes (mortality) each of which may constitute competing goals of the influenza immunization program. For example, vaccinating children, who tend to have higher contact rates than others, could result in a lower overall attack rate. However, as our results for the early initiation of the PC scenario showed, this strategy could leave the elderly (who experience higher mortality) relatively unprotected, thus increasing overall mortality.

In this study we included both symptomatic and asymptomatic infections in estimates of the overall attack rate. There are various estimates of the ratio of asymptomatic to symptomatic influenza cases in the literature [52,53]. More research should be directed towards conducting large-scale seroprevalence studies around the globe to reach a consensus on a plausible range corresponding to this ratio for $\mathrm{pH} 1 \mathrm{~N} 1$. When symptomatically infected, individuals may change their behaviour, deciding to stay home or cancel appointments, resulting in a reduction in their social contacts. Meanwhile, asymptomatically infected individuals may not observe such stringent self-isolation procedures but may also be less contagious owing to fewer projectile symptoms (i.e. coughing or sneezing). This effect was taken into account in the model and the related parameters were varied during sensitivity analyses. Similarly, other parameters that lack definitive parameterization in the public health literature (e.g., latent period, infectious periods) were included in the sensitivity analyses, to ensure the robustness of the reported results.

We assumed that during the herald wave in spring and early summer 2009, a relatively small fraction of the population was infected by pH1N1 symptomatically or asymptomatically. This assumption was supported by the marked difference in influenza activity in the province of $\mathrm{BC}$ between the two periods of April to August and September to December, based on both laboratoryconfirmed cases and physicians' visit counts (see Figure S8, Additional file 1). This pattern is in contrast with the attack rate reported in other geographic areas, such as England [43,44,47], where sizable pH1N1 activity was observed in June and July. In the latter case, before comparing various immunization strategies, adjustments should be made to the assumption on the number of remaining susceptible individuals at the beginning of the second wave.

We demonstrated that while vaccine efficacy is an important factor in the outcome of vaccination before or during the early stages of an epidemic, its impact on the overall attack rate diminishes significantly when the start of the campaign approaches or passes the epidemic peak-time. Simulation results suggest that when vaccination begins near the peak of the epidemic, a $50 \%$ efficacious vaccine may reduce the overall attack rate by only $5 \%$ less than a $100 \%$ efficacious vaccine. This result, along with our findings about the importance of vaccination timing, confirm the nostrum that no matter how effective a vaccine may be in theory, it must be administered in a timely fashion to have an effect on individual or herd immune protection.

True pH1N1 infection incidence is difficult to determine, as many cases go unreported, and an unknown fraction of $\mathrm{pH} 1 \mathrm{~N} 1$ cases are asymptomatic. To support our claim that our model predictions are consistent with the epidemic, we compared the age-distribution of reported, laboratory-confirmed $\mathrm{pH} 1 \mathrm{~N} 1$ cases in the GVRD (data from the BCCDC Laboratory and BC Ministry of Health) with the age distribution of infections predicted by our model (Figure 1). We found reasonable agreement between model predictions and reported cases in age-related trends.

Our results support, to a degree, the growing modeling literature claiming that the choice of vaccination strategy can have a substantial impact on the overall attack rate of pandemic influenza. This literature largely relies on careful, detailed modeling of age structure and/ or disease vulnerability levels (e.g. [32], and more recently $[33,38])$. The novelty we bring into this growing body of research is the incorporation of contact structure, in addition to age structure, as derived from the underlying GVRD contact network model. However, as in [37], these results also highlight the relatively greater importance of vaccination campaign timing and speed than prioritization scheme before or during the initial phase of an epidemic. Importantly, our model predicts a general equivalence of different prioritization schemes when vaccination begins at or beyond the epidemic peak.

Our results suggest that there can be two "best" targeting strategies: best given the specific vaccination 
campaign start time relative to the epidemic peak, and best overall given ignorance of the occurrence time of the epidemic peak (cf. Figure 4). Optimizing targeting strategies according to timing, age and disease vulnerability were carefully discussed in [18,34-36]. We leave the corresponding difficult optimization calculation using our model-which again, in contrast to previous work incorporates contact structure in addition to age structure-for future work. However we should comment that the PC strategy was chosen for comparison with the optimal strategy proposed in [18]; in there the PC strategy, when applied before the initiation of the epidemic, is the best choice in terms of both attack rate and mortality reduction. That our predictions differ may be in part due to the difference in assumptions on vaccine efficacy: while we assume equal efficacy across all age groups, Medlock et al. [18] assume that vaccines offer lesser protection in the elderly population, the population with the highest case mortality rates.

It should be noted that we assumed $100 \%$ coverage in our parents and children and parents and children/ actual sequence scenarios, which may be unrealistically high. We acknowledge that this exaggerates the apparent superiority of this approach, relative to the other strategies. However this strategy has a lower overall coverage (36\%) than the actual coverage or uniform coverage strategies $(47 \%)$. Given the success of the PC strategy in spite of its lower overall coverage, our results therefore suggest that, for campaigns initiated before the epidemic peak, it would be worthwhile for policy-makers to consider age-based vaccine targeting strategies assuming that high coverage rates are achievable in the targeted groups. The improvements in attack rate and mortality reduction offered by the $\mathrm{PC}+$ strategy, at equal coverage to $\mathrm{AC}$ and $\mathrm{UC}$, strengthen this suggestion.

In addition, it should be noted that our results apply to the pandemic scenario where a shift in the age distribution toward greater morbidity and mortality in younger age groups is a recognized hallmark compared to seasonal influenza [14]. Our results of superior reduction in mortality with the PC strategy administered during the early rise in a pandemic wave may not apply during seasonal campaigns when attack rates are much lower and thus population mortality due to influenza is much lower for children and adults but higher for the elderly, who remain at intrinsically higher risk of severe influenza outcomes if infected.

\section{Conclusion}

In circumstances in which vaccine production is delayed due to technological or logistical barriers, as seen with the $\mathrm{pH} 1 \mathrm{~N} 1$ vaccine, it is critical to have a good estimate of the timing of the epidemic peak before making policy decisions on vaccination strategies. Careful modeling may provide decision makers with estimates of these effects before the epidemic peak to motivate production efficiencies and inform policy decisions. Integration of real-time surveillance data with mathematical models is paramount to detect early upswings in illness activity heralding an epidemic peak and to enable public health to optimize the community benefits from proposed interventions before that occurs.

\section{Additional material}

\section{Additional file 1:}

\section{Acknowledgements}

The authors would like to acknowledge the support of the Canadian Institute of Health Research (CIHR) through the grant no. PTL-97126 to the Canadian Consortium for Pandemic Preparedness Modeling (CanPan). This research has been enabled by the use of computing resources provided by WestGrid and Compute/Calcul Canada. We would like to thank Robert Smith from the Population Health, Surveillance and Epidemiology Division, the British Columbia Ministry of Health for providing the physicians' billing data and summary of the BC Centre for Disease Control Virology Laboratory pH1N1 laboratory confirmation. Authors recognize local health care providers and public health practitioners for their invaluable contribution to pandemic H1N1 surveillance in British Columbia and Travis Hottes and Naveed Janjua of the BC Centre for Disease Control Influenza Team for their analysis and summary of that.

\section{Author details}

${ }^{1}$ Division of Mathematical Modeling, University of British Columbia Centre for Disease Control, 655 West 12th Avenue, V5Z 4R4 Vancouver, British Columbia, Canada. ${ }^{2}$ Department of Mathematics, University of British Columbia, Vancouver, British Columbia, Canada. ${ }^{3}$ Dalla Lana School of Public Health, University of Toronto, Toronto, Ontario, Canada. ${ }^{4}$ Departments of Public Health and Medicine, Weill Medical College of Cornell University, New York, NY, USA. ${ }^{5}$ New York-Presbyterian Hospital, New York, NY, USA.

${ }^{6}$ Department of Mathematics and Statistics, University of Victoria, Victoria, British Columbia, Canada. ${ }^{7}$ Section of Integrative Biology, The University of Texas at Austin, Austin, TX, USA. ${ }^{8}$ Department of Pathology and Molecular Medicine, McMaster University, Hamilton, Ontario, Canada. ${ }^{9}$ Centre for Communicable Diseases and Infection Control, Public Health Agency of Canada, Toronto, Ontario, Canada. ${ }^{10}$ Epidemiology Services, British Columbia Centre for Disease Control, Vancouver, British Columbia, Canada.

${ }^{11}$ Surveillance Lab, Department of Epidemiology and Biostatistics, McGill University, Montreal, Québec, Canada. ${ }^{12}$ Bureau de surveillance épidémiologique, Direction de santé publique de Montréal, Montréal, Québec, Canada. ${ }^{13}$ Institute for Clinical Evaluative Sciences, Toronto, Ontario, Canada. ${ }^{14}$ Centre for Disease Modelling, York University, Toronto, Ontario, Canada. ${ }^{15} \mathrm{~S} c h o o l$ of Population and Public Health, Faculty of Medicine, University of British Columbia, Vancouver, Canada.

\section{Authors' contributions}

Conception and design of the study: CJM, TA, FD, HN, PB; Execution of mathematical simulations: CJM, TA, MR; Draft of the manuscript and supplementary material: CJM, TA, FD, HN, PB; Analysis of BC Health data: CJM, MR, DB, EK, SD, BRC, PB; Building Vancouver Contact Network: DB, EK, PB; Verification and validation of the mathematical modeling framework: CJM, MR, PvdD, BF, MJ, ML, GA, WJ, MS, CD, PB; Providing clinical and public health insight into analyzing the results: FD, HN, SM, SD, BD, KJ, BR; Providing critical input on various drafts of the manuscript: All Authors.

\section{Competing interests}

The authors declare that they have no competing interests. 
Received: 31 March 2011 Accepted: 14 December 2011

Published: 14 December 2011

\section{References}

1. Chan M: World now at the start of 2009 influenza pandemic.[http://www. who.int/mediacentre/news/statements/2009/ h1n1_pandemic_phase6_20090611/en/].

2. CDC 2010-2011 Influenza season week 38 ending September 24, 2011, U.S. Virologic Surveillance. [http://www.cdc.gov/flu/weekly/].

3. WHO, Successes and challenges of 2009. [http://www.who.int/ mediacentre/multimedia/podcasts/2009/year_end_20091230/en/].

4. British Columbia influenza surveillance bulletin 2009-10: number 12 , week 51, December 20-26, 2009. [http://www.bccdc.ca/NR/rdonlyres/ 1AE2F127-4102-4C1A-9610-8519AE979AE7/0/ InfluBulletin Number12 Week51 0910.pdf].

5. National Advisory Committee on Immunization (NACl): Statement on influenza vaccination for the 2008-2009 season. An Advisory Committee Statement (ACS). Can Commun Dis Rep 2008, 34(ACS-3):1-46.

6. Fisman DN, Savage R, Gubbay J, Achonu C, Akwar H, Farrell DJ, Crowcroft NS, Jackson P: Older age and a reduced likelihood of 2009 H1N1 virus infection. N Engl J Med 2009, 361(20):2000-2001.

7. Centers for Disease Control and Prevention (CDC): Update: novel influenza A (H1N1) virus infection - Mexico, March-May, 2009. MMWR Morb Mortal Wkly Rep 2009, 58(21):585-589.

8. Fraser C, Donnelly CA, Cauchemez S, Hanage WP, Van Kerkhove MD, Hollingsworth TD, Griffin J, Baggaley RF, Jenkins HE, Lyons EJ, Jombart T, Hinsley WR, Grassly NC, Balloux F, Ghani AC, Ferguson NM, Rambaut A, Pybus OG, Lopez-Gatell H, Apluche-Aranda CM, Chapela IB, Zavala EP, Guevara DM, Checchi F, Garcia E, Hugonnet S, Roth C: The WHO rapid pandemic assessment collaboration: pandemic potential of a strain of influenza A (H1N1): Early Findings. Science 2009.

9. Centers for Disease Control and Prevention (CDC): 2009 pandemic influenza A (H1N1) virus infections - Chicago, Illinois, April-July 2009. MMWR Morb Mortal Wkly Rep 2009, 58(33):913-918.

10. Baker MG, Wilson N, Huang QS, Paine S, Lopez L, Bandaranayake D, Tobias M, Mason K, Mackereth GF, Jacobs M, Thornley C, Roberts S, McArthur C: Pandemic influenza A(H1N1)v in New Zealand: the experience from April to August 2009. Euro Surveill 2009, 14(34):19319.

11. Nishiura H, Castillo-Chavez C, Safan M, Chowell G: Transmission potential of the new influenza $\mathrm{A}(\mathrm{H} 1 \mathrm{~N} 1)$ virus and its age-specificity in Japan. Euro Surveill 2009, 14(22):19227.

12. National Center for Immunization and Respiratory Diseases, CDC, Centers for Disease Control and Prevention (CDC): Use of influenza A (H1N1) 2009 monovalent vaccine: recommendations of the Advisory Committee on Immunization Practices (ACIP), 2009. MMWR Recomm Rep 2009, 58(RR10):1-8.

13. Centers for Disease Control and Prevention (CDC): Serum cross-reactive antibody response to a novel influenza $A(\mathrm{H} 1 \mathrm{~N} 1)$ virus after vaccination with seasonal influenza vaccine. MMWR Morb Mortal Wkly Rep 2009, 58(19):521-524.

14. Bansal S, Pourbohloul B, Hupert N, Grenfell B, Meyers LA: The shifting demographic landscape of pandemic influenza. PLoS One 2010, 5(2): e9360.

15. Bansal S, Pourbohloul B, Meyers LA: A comparative analysis of influenza vaccination programs. PLoS Med 2006, 3(10):e387.

16. Halloran ME, Longini IM Jr: Public health. Community studies for vaccinating schoolchildren against influenza. Science 2006, 311(5761):615-616

17. Galvani AP, Reluga TC, Chapman GB: Long-standing influenza vaccination policy is in accord with individual self-interest but not with the utilitarian optimum. Proc Natl Acad Sci USA 2007, 104(13):5692-5697.

18. Medlock J, Galvani AP: Optimizing influenza vaccine distribution. Science 2009, 325(5948):1705-1708.

19. Kwong JC, Stukel TA, Lim J, McGeer AJ, Upshur RE, Johansen H, Sambell C, Thompson WW, Thiruchelvam D, Marra F, Svenson LW, Manuel DG: The effect of universal influenza immunization on mortality and health care use. PloS Med 2008, 5(10):e211.

20. Louie JK, Acosta M, Winter K, Jean C, Gavali S, Schechter R, Vugia D, Harriman K, Matyas B, Glaser CA, Samuel MC, Rosenberg J, Talarico J, Hatch D, California Pandemic (H1N1) Working Group: Factors associated with death or hospitalization due to pandemic 2009 influenza $A(H 1 N 1)$ infection in California. JAMA 2009, 302(17):1896-1902.

21. Vaillant $L$, La Ruche G, Tarantola A, Barboza P, epidemic intelligence team at InVS: Epidemiology of fatal cases associated with pandemic H1N1 influenza 2009. Euro Surveill 2009, 14(33):19309.

22. Tuite AR, Greer AL, Whelan M, Winter AL, Lee B, Yan P, Wu J, Moghadas S, Buckeridge D, Pourbohloul B, Fisman DN: Estimated epidemiologic parameters and morbidity associated with pandemic H1N1 influenza. CMAJ 2010, 182(2):131-136.

23. WHO recommendations on pandemic (H1N1) 2009 vaccines. [http:// www.who.int/csr/disease/swineflu/notes/h1n1_vaccine_20090713/en/index. html].

24. Public health agency of Canada guidance on H1N1 flu vaccine sequencing. [http://www.phac-aspc.gc.ca/alert-alerte/h1n1/vacc/vacc-eng. php].

25. Department of health priority groups for the vaccination program [http://www.dh.gov.uk/en/Publichealth/Flu/Swineflu/ InformationandGuidance/Naccinationprogramme/DH_105455/.

26. Skowronski DM, Hottes TS, McElhaney J, et al: Immuno-epidemiologic correlates of pandemic H1N1 surveillance observations: higher antibody and lower cell-mediated immune responses with advanced age. J Infect Dis 2010.

27. Pourbohloul B, Ahued A, Davoudi B, Meza R, Meyers LA, Skowronski DM, Villasenor I, Galvan F, Cravioto P, Earn DJ, Dushoff J, Fisman D, Edmunds WJ, Hupert N, Scarpino SV, Trujillo J, Lutzow M, Morales J, Contreras A, Chavez C, Patrick DM, Brunham RC: Initial human transmission dynamics of the pandemic (H1N1) 2009 virus in North America. Influenza Other Respi Viruses 2009, 3(5):215-222.

28. Yang Y, Sugimoto JD, Halloran ME, Basta NE, Chao DL, Matrajt L, Potter G,

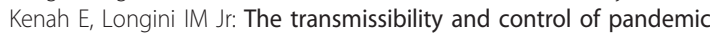
influenza A (H1N1) virus. Science 2009, 326(5953):729-733.

29. Gojovic MZ, Sander B, Fisman D, Krahn MD, Bauch CT: Modelling mitigation strategies for pandemic (H1N1) 2009. CMAJ 2009, 181(10):673-680.

30. Chowell G, Viboud C, Wang X, Bertozzi SM, Miller MA: Adaptive vaccination strategies to mitigate pandemic influenza: Mexico as a case study. PLoS One 2009, 4(12):e8164.

31. Moghadas SM, Bowman CS, Rost G, Fisman DN, Wu J: Post-exposure prophylaxis during pandemic outbreaks. BMC Med 2009, 7(1):73

32. Longini IM, Ackerman $E$, Elveback LR: An optimization model for influenza A epidemics. 1978, 38(1-2):141

33. Dushoff J, Plotkin JB, Viboud C, Simonsen L, Miller M, Loeb M, Earn DJ: Vaccinating to protect a vulnerable subpopulation. PLoS Med 2007, 4(5): e174.

34. Wallinga J, van Boven M, Lipsitch M: Optimizing infectious disease interventions during an emerging epidemic. Proc Natl Acad Sci USA 2010, 107(2):923-928.

35. Keeling MJ, White PJ: Targeting vaccination against novel infections: risk, age and spatial structure for pandemic influenza in Great Britain. J R SOC Interface 2011, 8(58):661-670.

36. Mylius SD, Hagenaars TJ, Lugner AK, Wallinga J: Optimal allocation of pandemic influenza vaccine depends on age, risk and timing. Vaccine 2008, 26(29-30):3742-3749.

37. Knipl DH, Rost G: Modelling the strategies for age specific vaccination scheduling during influenza pandemic outbreaks. Math Biosci Eng 2011, 8(1):123-139.

38. Pourbohloul B, Meyers LA, Skowronski DM, Krajden M, Patrick DM, Brunham RC: Modeling control strategies of respiratory pathogens. Emerg Infect Dis 2005, 11(8):1249-1256.

39. Meyers LA, Pourbohloul B, Newman ME, Skowronski DM, Brunham RC: Network theory and SARS: predicting outbreak diversity. J Theor Biol 2005, 232(1):71-81

40. White LF, Wallinga J, Finelli L, Reed C, Riley S, Lipsitch M, Pagano M: Estimation of the reproductive number and the serial interval in early phase of the 2009 influenza A/H1N1 pandemic in the USA. Influenza Other Respi Viruses 2009, 3(6):267-276.

41. British Columbia Influenza Surveillance Bulletin 2008-09: Number 32, Week 36, September 6-12, 2009. [http://www.bccdc.ca/NR/rdonlyres/ 69C1F336-97CD-4D97-8CE9-C67757294E62/0/ InfluBulletin_Number32_Week36_0809.pdf]. 
42. Statistics Canada 2006 Community Profiles. [http://www12.statcan.ca/ census-recensement/2006/dp-pd/prof/92-591/index.cfm?Lang=E].

43. Liao CM, Yang SC, Chio CP, Chen SC: Understanding influenza virusspecific epidemiological properties by analysis of experimental human infections. Epidemiol Infect 2010, 138(6):825-835.

44. De Serres G, Rouleau I, Hamelin ME, Quach C, Skowronski D, Flamand L, Boulianne N, Li Y, Carbonneau J, Bourgault A, Couillard M, Charest H, Boivin G: Contagious period for pandemic (H1N1) 2009. Emerg Infect Dis 2010, 16(5):783-788.

45. Product Information Leaflet, Arepanrix ${ }^{\mathrm{TM}}$ H1N1. [http://www.gsk.ca/ english/docs-pdf/Arepanrix_2010.pdf].

46. Waddington CS, Walker WT, Oeser C, Reiner A, John T, Wilkins S, Casey M, Eccleston PE, Allen RJ, Okike I, Ladhani S, Sheasby E, Hoschler K, Andrews N, Waight P, Collinson AC, Heath PT, Finn A, Faust SN, Snape MD, Miller E, Pollard AJ: Safety and immunogenicity of AS03B adjuvanted split virion versus non-adjuvanted whole virion $\mathrm{H} 1 \mathrm{~N} 1$ influenza vaccine in UK children aged 6 months- 12 years: open label, randomised, parallel group, multicentre study. BMJ 2010, 340:с2649.

47. Donaldson L, Rutter PD, Ellis BM, Greaves FE, Mytton OT, Pebody RG, Yardley IE: Mortality from pandemic A/H1N1 2009 influenza in England: public health surveillance study. BMJ 2009, 339:b5213.

48. Skowronski DM, Janjua NZ, De Serres G, Hottes TS, Dickinson JA, Crowcroft N, Kwindt TL, Tang P, Charest H, Fonseca K, Gubbay JB, Bastien N, Li Y, Petric M: Effectiveness of AS03 adjuvanted pandemic H1N1 vaccine: case-control evaluation based on sentinel surveillance system in Canada, autumn 2009. BMJ 2011, 342:C7297.

49. Clark TW, Pareek M, Hoschler K, Dillon H, Nicholson KG, Groth N, Stephenson I: Trial of 2009 influenza A (H1N1) monovalent MF59adjuvanted vaccine. N Engl J Med 2009, 361(25):2424-2435.

50. Van Buynder PG, Dhaliwal JK, Van Buynder JL, Couturier C, MinvilleLeblanc M, Garceau R, Tremblay FW: Protective effect of single-dose adjuvanted pandemic influenza vaccine in children. Influenza Other Respi Viruses 2010, 4(4):171-178.

51. Baguelin M, Hoek AJ, Jit M, Flasche S, White PJ, Edmunds WJ: Vaccination against pandemic influenza A/H1N1v in England: a real-time economic evaluation. Vaccine 2010, 28(12):2370-2384.

52. Miller MA, Viboud C, Balinska M, Simonsen L: The signature features of influenza pandemics-implications for policy. N Engl J Med 2009, 360(25):2595-2598

53. Hadler JL, Konty K, McVeigh KH, Fine A, Eisenhower D, Kerker B, Thorpe L: Case fatality rates based on population estimates of influenza-like illness due to novel H1N1 influenza: New York City, May-June 2009. PLoS One 2010, 5(7):e11677.

54. Towers S, Feng Z: Pandemic H1N1 influenza: predicting the course of a pandemic and assessing the efficacy of the planned vaccination program in the United States. Eurosurveillance 2009, 14:19358.

55. Cutler J, Schleihauf E, Hatchette TF, Billard B, Watson-Creed G, Davidson R, Li Y, Bastien N, Sarwal S, the Nova Scotia Human Swine Influenza Investigation Team: Investigation of the first cases of human to-human infection with the new swine-origin influenza A (H1N1) virus in Canada. CMAJ 2009, 181:159-163.

\section{Pre-publication history}

The pre-publication history for this paper can be accessed here: http://www.biomedcentral.com/1471-2458/11/932/prepub

doi:10.1186/1471-2458-11-932

Cite this article as: Conway et al:: Vaccination against 2009 pandemic $\mathrm{H} 1 \mathrm{~N} 1$ in a population dynamical model of Vancouver, Canada: timing is everything. BMC Public Health 2011 11:932.

\section{Submit your next manuscript to BioMed Central and take full advantage of:}

- Convenient online submission

- Thorough peer review

- No space constraints or color figure charges

- Immediate publication on acceptance

- Inclusion in PubMed, CAS, Scopus and Google Scholar

- Research which is freely available for redistribution

Submit your manuscript at www.biomedcentral.com/submit
Biomed Central 